\title{
Effects of Different Heating Methods on Positional Differences in Taste, Texture, Color, and Palatability of Simmered Food: Comparison of Sensor Responses and Sensory Evaluation Results
}

\author{
Yuko Fujimoto*, Hiroko Chiba ${ }^{1}$, Ryo Okawa and Kiyoshi Toko ${ }^{1}$ \\ Energy Engineering Dept., Osaka Gas Co., Ltd., \\ 5-11-61 Torishima, Konohana-ku, Osaka-shi, Osaka 554-0051, Japan \\ ${ }^{1}$ Graduate School of Information Science and Electrical Engineering, Kyushu University, \\ 744 Motooka, Nishi-ku, Fukuoka-shi, Fukuoka 819-0395, Japan
}

(Received December 24, 2014; accepted May 8, 2015)

Key words: taste sensor, texture analysis, color difference analysis, sensory evaluation, palatability

The palatability of food depends on various factors including the five human senses. The palatability of beverages can now be objectively and quantitatively evaluated owing to the development of various sensing devices including taste sensors. In this study, we carried out a sensory evaluation and physicochemical analyses using three sensing devices (a taste sensor, a creep meter, and a colorimeter) to objectively evaluate the palatability of foods simmered by different heating methods. The samples used for the evaluation were selected from daikon radishes simmered in large quantities using professional heating appliances. The results of the sensory evaluation were in good agreement with those of the analysis using each sensing device. It was clarified that the palatability, taste, texture, and color of the simmered food (daikon) placed at different positions (outer and middle regions of a pan) were affected by the heating method.

\section{Introduction}

The palatability of foods depends on various factors including the five human senses. Sensors and measurement systems related to the senses of touch, vision, and hearing have already been developed, whereas the development of those related to taste and odor has lagged behind. Sensors related to touch, sight, and hearing can be realized by measuring the corresponding quantities, which can be quantified mainly using semiconductor devices. In contrast, taste and odor sensors (i.e., electronic tongues and noses) are required to detect many chemical substances and obtain output information

*Corresponding author: e-mail: yuko-kimura@osakagas.co.jp 
on the taste and odor perceived by humans. Suitable sensor materials for receiving such chemical substances have not been clarified. Recently, considerable progress has been made in the development of taste and odor sensors, and taste sensors are already in practical use. ${ }^{(1-8)}$ Odor sensors based on various materials and principles have also been proposed, some of which have been practically applied.(1,9-12)

Thus, various sensing devices that can be used to objectively evaluate the palatability of beverages, in which palatability is dominantly determined by taste, are being developed. However, there have been no reports on the simultaneous contribution of three or more elements, such as taste (perceived by the sense of taste), texture (perceived by touch), and color (perceived by sight), to palatability, to the best of our knowledge. In this study, we focused on simmered food, whose palatability is considered to be perceived by multiple senses, as a first step and performed a sensory evaluation and physicochemical analyses using different sensing devices to objectively evaluate the palatability of simmered food.

In commercial kitchens, large quantities of food are cooked to provide customers with food products, and hence, cooking with high uniformity and repeatability in terms of taste and color must be possible. A report on home cooking showed that the difference in heating mechanism between a gas cooking stove and an induction heating (IH) cooker resulted in differences in the convection and boiling properties of water in a pan. ${ }^{(13)}$

Previously, we reported that the difference in the heating method affected the color, texture, and taste of simmered food that was cooked in a large quantity, similarly to the case of cooking in commercial kitchens. ${ }^{(14)}$ In this study, we focused on daikon radishes as simmered food, in a similar way to the previous study, ${ }^{(14)}$ and examined the differences in the taste and texture of half-disk daikon samples placed in the outer and middle regions of a pan. If the taste and texture of samples placed at different positions are different, the cooking method is considered to have low uniformity that results in variations in taste and texture. In this study, we examined the effects of different heating methods on the taste and texture of simmered food (half-disk pieces of daikon) placed at different positions in a pan by comparing the results of objective evaluations using sensing devices with those of a sensory evaluation.

\section{Materials and Methods}

\subsection{Materials}

As typical heating appliances, a professional tabletop gas stove (Ozaki Co., Ltd.; rated gas consumption, $0.47 \mathrm{~m}^{3} / \mathrm{h}$ ) and an $\mathrm{IH}$ cooker (Nichiwa Electric Corporation; rated power consumption, $2.5 \mathrm{~kW}$ ) were used for cooking. Both cookers are general appliances used for heating and cooking. A professional stainless-steel pan (diameter, $27 \mathrm{~cm}$ ) with a handle and suitable for IH was used. The surface temperature of the pan during heating over each cooker was visualized using a thermal imager (NEC San-ei Instruments, Ltd., TH9100WLN). ${ }^{(14)}$ The results were in agreement with those reported by Kawakami et al.;(15) the entire pan surface, including the bottom and side, was heated by the gas stove, whereas only part of the bottom of the pan over the coil was heated by the IH cooker. ${ }^{(14)}$ On the basis of these observations, the heating methods using the gas 
and IH cookers are referred to as "pan surface heating" and "local heating", respectively.

We adopted simmering as the cooking method considering that the variation in the surface temperature of the pan during heating and physical phenomena such as convection and boiling are reflected in cooked products. The food material was daikon because its white color was ideal for confirming changes in color due to cooking and because the light taste of the food itself makes it ideal for comparing the taste among samples. The daikon was simmered in commercial liquid soup stock (Kikkoman Corporation, concentrated liquid bonito soup stock) diluted fivefold (volume ratio).

\subsection{Cooking method}

The cooking and heating conditions were as follows.

\subsubsection{Cooking conditions}

We tried to set the cooking conditions as equal as possible in order to compare them fairly. A daikon was cut into a disk shape with a diameter of $6 \mathrm{~cm}$ and a height of $3.5 \mathrm{~cm}$ using a stainless-steel die. The stem end of the daikon was excluded from the samples. The disk-shaped piece was cut into two equal half-disk pieces; one was used for pan surface heating and the other was used for local heating. The half-disk pieces were placed at the same position and in the same direction in the pan. As shown in Fig. 1, 25 pieces of daikon (outer, 12; middle, 8 ; inner, 5) were placed at the bottom of the pan and were stacked in three levels inside the pan. The amount of soup stock in the pan was $1500 \mathrm{~g}$ and its initial temperature was $20^{\circ} \mathrm{C}$.

\subsubsection{Heating conditions}

In accordance with a general simmering method, the daikon samples were heated over medium heat for $15 \mathrm{~min}$ until the soup boiled, then heated over low heat for $30 \mathrm{~min}$, and left to stand for $30 \mathrm{~min}$ to cool the samples; this was defined as one cooking cycle. The heating conditions of the two heating methods were standardized so that (1) the

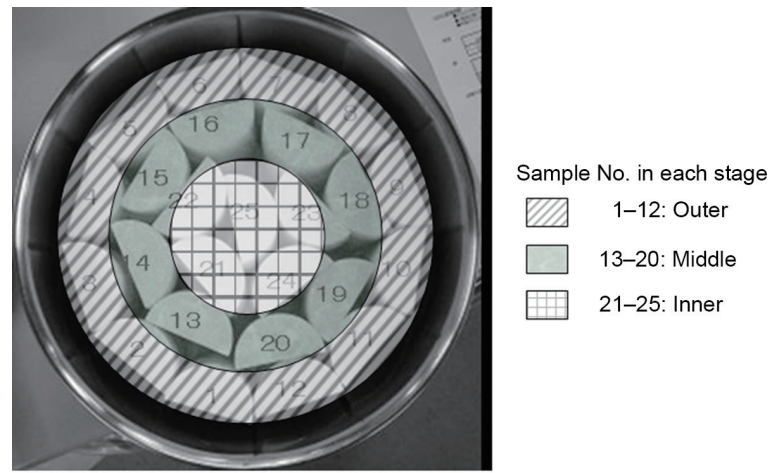

Fig. 1. Daikon samples placed in pan. 
amount of evaporation was $465 \mathrm{~g}$ in the case of $3600 \mathrm{~g}$ daikon samples and $1500 \mathrm{~g}$ soup stock, (2) the rate of increase in the temperature of the stock in the pan over medium heat was in the range of $20-70{ }^{\circ} \mathrm{C}$ (this is because evaporation starts to occur when the temperature exceeds $70{ }^{\circ} \mathrm{C}$ ), and (3) the amount of evaporation in the pan over low heat was the same.

\subsection{Sensory evaluation and physicochemical analyses}

The samples placed in the upper and middle levels in the pan became mushy upon heating. Therefore, these samples were excluded and only the samples placed in the lower level were used for evaluation. Moreover, the number of samples placed in the inner region was insufficient. Hence, only the samples placed in the outer and middle regions were used for comparison.

Figure 2 shows images of samples. Some were charred and excluded from the samples for sensory evaluation and physiochemical analyses.

\subsubsection{Sensory evaluation}

A sensory evaluation test was carried out as below to examine the taste, texture, color, and palatability of the samples subjected to pan surface heating and local heating. In accordance with the Declaration of Helsinki, we sufficiently explained the contents of the test and obtained consent from female subjects in their $20 \mathrm{~s}-40 \mathrm{~s}(N=44)$ in advance. The sensory evaluation was performed by $7-10$ subjects per day over five days. Here, the subjects were selected from among those with a score greater than or equal to the average in a five-taste test conducted in advance in order to avoid the difference among people and the unreliability of the results. The subjects were requested to evaluate, on a nine-point scale, a total of four samples placed in the outer and middle regions of the pan during pan surface heating and local heating.

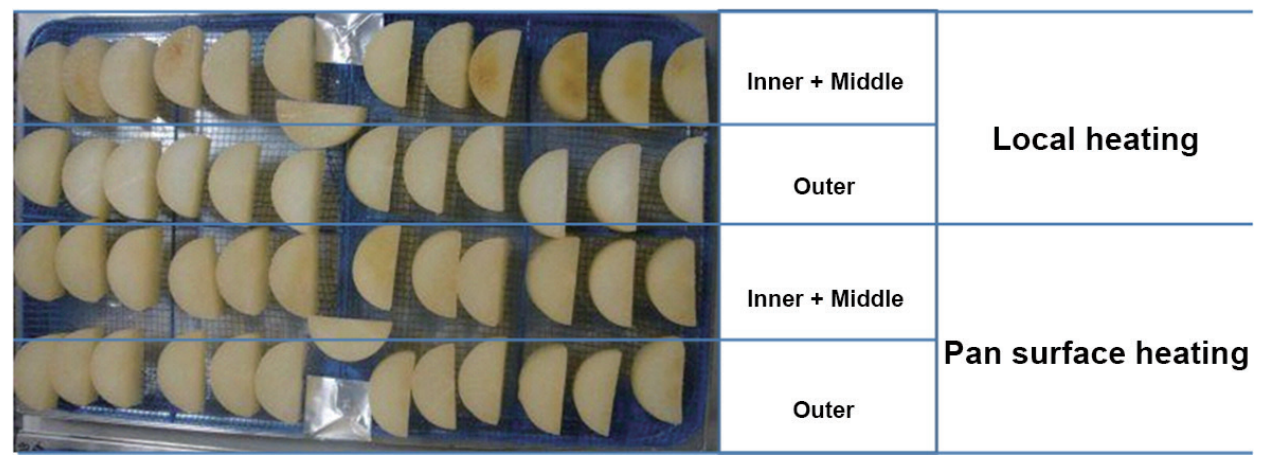

Fig. 2. Images of daikon samples. 


\subsubsection{Physicochemical analyses}

In addition, three physicochemical analyses were performed to examine the taste, texture, and color of the samples. As for the odor, there is no reliable odor sensor at present, and hence we did not evaluate the odor in this study.

\subsubsection{Analysis of taste using taste sensor}

The intensity of the taste of the samples was measured using a taste sensor (Taste Sensing System TS-5000Z, Intelligent Sensor Technology, Inc.) to quantify saltiness, $k o k u$, and umami. While koku or kokumi is generally defined as a taste with mouthfulness and continuity, ${ }^{(16,17)}$ it was quantified using the taste sensor on the basis of the measured change in the membrane potential caused by adsorption (CPA) of chemical substances responsible for umami. CPA corresponds to the so-called aftertaste. ${ }^{(1-4)}$ Although lipid/polymer membranes of the taste sensor respond to several taste substances simultaneously, the CPA value is specific to adsorptive taste substances. Recent studies show that there is high correlation between the CPA value and the amount of substances adsorbed onto the lipid/polymer membranes. ${ }^{(18,19)}$ Koku or kokumi can be evaluated using the CPA value of the umami sensor electrode of the taste sensor. ${ }^{(3,20)}$ Each simmered daikon sample was crushed for 1 min using a mixer, diluted twofold with roomtemperature pure water, stirred, and filtered through gauze to obtain a sample solution to be used in the analysis. Each sample solution was analyzed three times and the average of the three measurements was used in the evaluation.

\subsubsection{Analysis of texture by breaking stress measurement}

Six cubic pieces with 1.5 -cm-long sides were obtained from each sample and their texture was analyzed using a creep meter (RE2-33005B, Yamaden Co., Ltd.). A toothlike wedge-shaped resin plunger (No. 49, W13 $\times 30^{\circ}$ edge, 1 -mm-wide, planar-wedge shape, Yamaden Co., Ltd.) was used. Breaking stress was measured at a storage pitch of $0.1 \mathrm{~s}$ and a sample table speed of $1 \mathrm{~mm} / \mathrm{s}$. Three samples obtained from the outer region and two samples obtained from the middle region were analyzed for both pan surface heating and local heating.

\subsubsection{Analysis of color using colorimeter}

The color of the samples was analyzed using a spectrophotometer (CM-5, Konica Minolta, Inc.). Each sample was cut widthwise into two. Redness ( $a$-value), yellowness ( $b$-value), and brightness ( $L$-value) were measured at a total of ten positions (surface, five; internal, five). A total of six samples (outer region, three; middle region, three) were used for both pan surface heating and local heating.

\section{Results and Discussion}

\subsection{Sensory evaluation}

Figure 3 shows the positional difference in each index obtained in the sensory evaluation. The average of the evaluation scores given by the panelists was calculated for each index. Color intensity, hardness, flavor intensity, and koku did not show any 


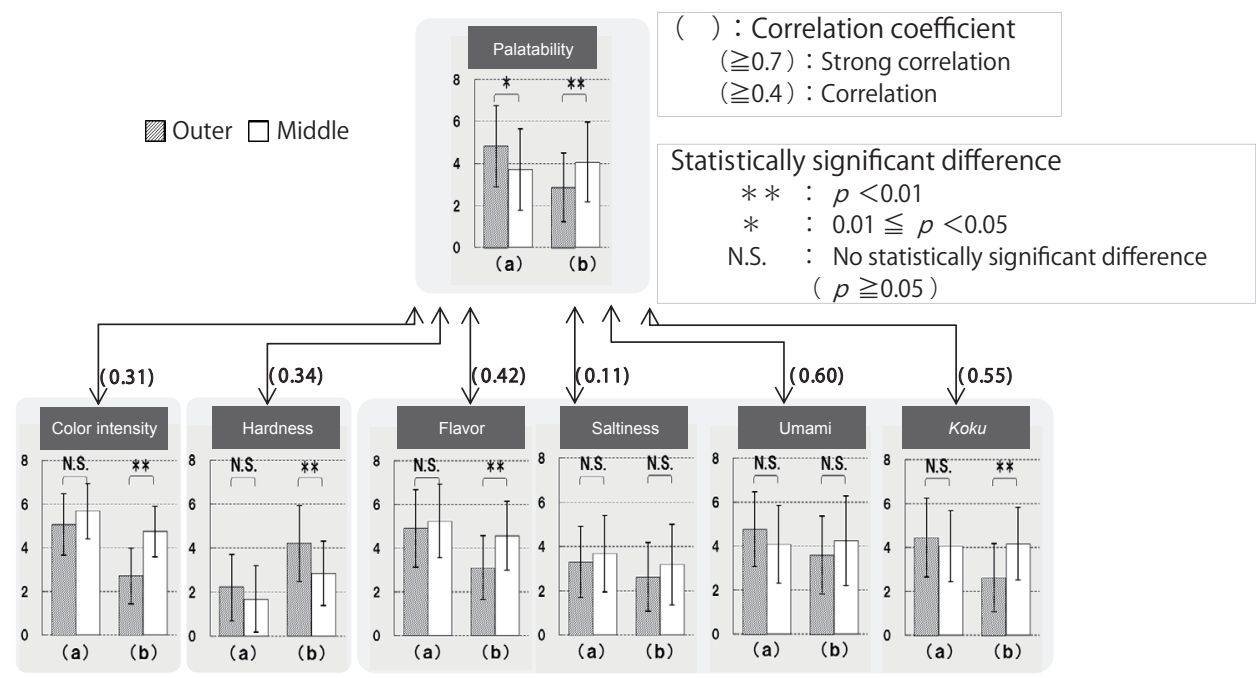

Fig. 3. Results of sensory evaluation. (a) Pan surface heating. (b) Local heating.

difference between the outer and middle regions (hereafter, simply denoted as "positional difference") for pan surface heating ( $p \geq 0.05$ ) (a). In contrast, these indices showed a statistically significant positional difference for local heating $(p<0.01)(b)$. Saltiness and umami showed no positional difference for pan surface heating or local heating. Palatability showed a positional difference $(0.01 \leq p<0.05)$ for pan surface heating and a statistically significant positional difference for $(p<0.01)$ local heating.

Palatability is considered to involve color intensity, hardness, flavor intensity, saltiness, umami, and koku. Among these indices, umami had the largest correlation coefficient $(r=0.60)$, followed by koku $(r=0.55)$, flavor intensity $(r=0.42)$, hardness $(r=0.34)$, color intensity $(r=0.31)$, and saltiness $(r=0.11)$, as shown in Fig. 3. This indicates that the palatability is strongly affected by the indices related to taste, followed by texture and color.

\subsection{Taste}

Figure 4 shows the measured values for saltiness, umami, and koku obtained using the taste sensor. These values mean the objective expression using the scale of taste, which is obtained by linear transformation of sensor outputs. ${ }^{(1-3)}$ As shown in Fig. 4, the positional difference in saltiness is statistically significant for both (a) pan surface heating and (b) local heating. Umami and koku showed no positional difference for either heating method.

For umami, the results obtained using the taste sensor were in excellent agreement with those of the sensory evaluation. For saltiness, the taste sensor detected slight differences that could not be perceived by the panelists in the cases of both pan surface 

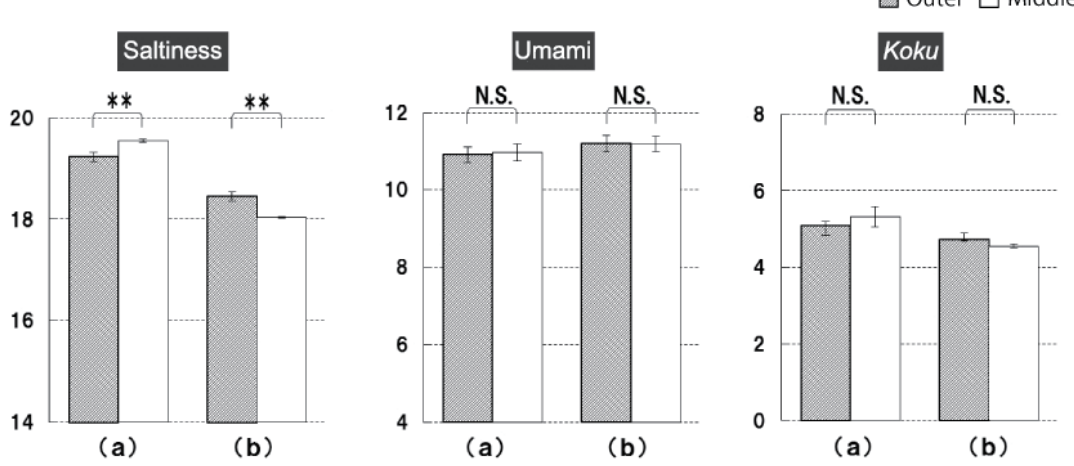

Fig. 4. Results of analysis using taste sensor. (a) Pan surface heating. (b) Local heating.

heating and local heating; hence, the results obtained using the taste sensor were different from those of the sensory evaluation.

For koku, positional differences were not observed for either heating method in the results obtained using the taste sensor but were observed for local heating in the results of the sensory evaluation in Fig. 3. This may be because the significant positional difference in color intensity in Fig. 3 affects the results of the sensory evaluation regarding $k o k u$, as reported by Ito et al.(21) Namely, visual information affected the sense of taste of humans.

\subsection{Texture}

Figure 5 shows the analysis results of hardness as a measure of texture. The positional difference in local heating (b) was greater than that in pan surface heating (a). In particular, the samples placed in the outer region and subjected to local heating (b) were harder than the other three samples. Moreover, the stress of these samples was still high after reaching the breaking stress, suggesting that the inside of the samples was also hard. These results were in good agreement with those of the sensory evaluation shown in Fig. 3.

To discuss the analysis results in more detail, we focused on the positional differences at strains of $18 \%$ (corresponding to the maximum stress) and 50\%, as shown in Fig. 6. For pan surface heating, a positional difference was observed at a strain of $18 \%$ but not at a strain of $50 \%$. For local heating, a statistically significant positional difference $(p<0.01)$ was observed at strains of both 18 and $50 \%$.

\subsection{Color}

Figure 7 shows the analysis results for the color of the samples. For pan surface heating (a), there were no positional differences in redness ( $a$-value), yellowness ( $b$-value), and brightness ( $L$-value). For local heating (b), a positional difference was not observed in the $b$-value but was observed in the $a$ - and $L$-values. The finding that the positional 


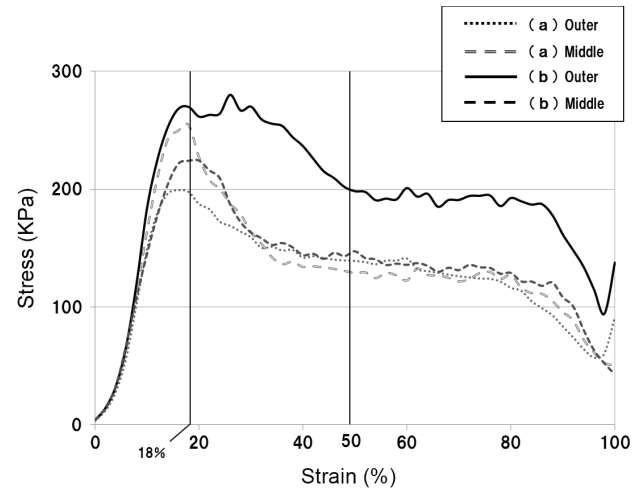

Fig. 5. Results of texture analysis. (a) Pan surface heating. (b) Local heating.
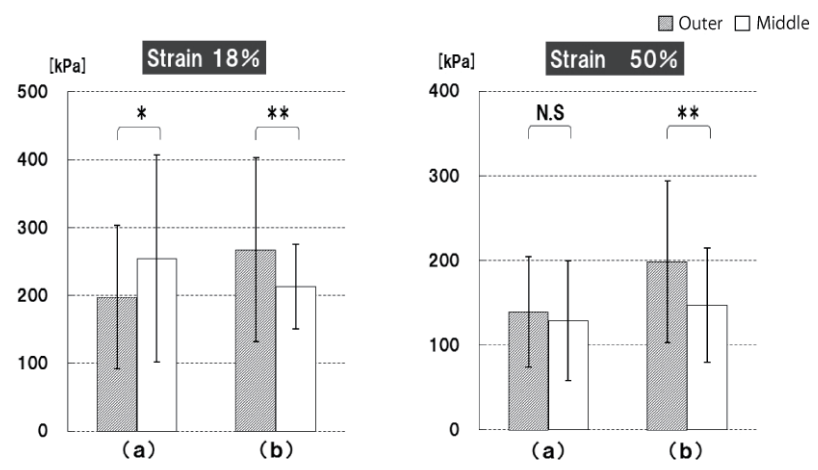

Fig. 6. Breaking stress for different strains. (a) Pan surface heating. (b) Local heating.

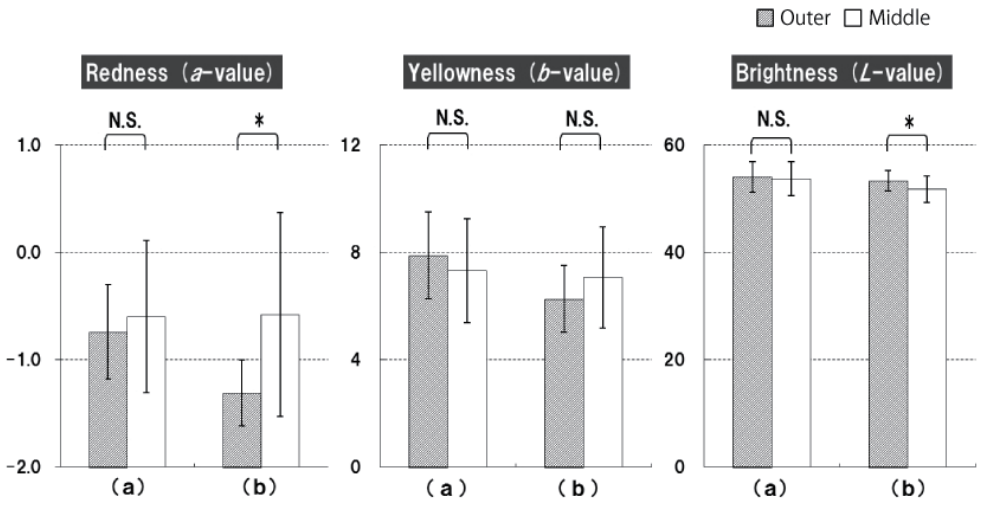

Fig. 7. Results of color difference analysis. (a) Pan surface heating. (b) Local heating. 
difference in color was not observed for pan surface heating but was observed for local heating is in agreement with the results of the sensory evaluation in Fig. 3.

Out of a total of 19 cooking trials, charred samples, which were excluded from the evaluation targets, were found in only one of the trials for the samples subjected to pan surface heating (the occurrence of charred samples was 5\%). However, at least one charred sample was found in all the trials for the samples subjected to local heating, meaning that the occurrence of charred samples was $100 \%$. Therefore, the positional difference among the samples, including those excluded from the evaluation targets, was greater for local heating than for pan surface heating.

\subsection{Correlation between palatability and output from sensors}

As discussed above, the positional differences (outer and middle regions) in the three elements of the palatability of simmered food, i.e., taste (perceived by taste sense), texture (perceived by tactile sense), and color (perceived by visual sense), were compared between the sensory evaluation and physicochemical analyses using sensing devices. The two results were in good agreement. Moreover, the correlation between palatability and each index measured using the sensing devices was examined as follows. For taste, the values for umami, koku, and saltiness obtained using the taste sensor (Fig. 4) were adopted. For texture, the breaking stress at a strain of $50 \%$ (Fig. 6) was adopted. For color, the $a-, b$-, and $L$-values (Fig. 7) were adopted. The coefficients of correlation were 0.65 (umami), 0.30 (koku), 0.29 (saltiness), 0.75 (breaking stress at a strain of $50 \%$ ), 0.69 ( $a$-value), 0.94 ( $b$-value), and 0.11 ( $L$-value).

The following findings can be obtained from the above results. When the values obtained using the sensing devices were focused on, the element that most strongly correlated with palatability was color, which was followed by texture and taste. In contrast, when the values obtained in the sensory evaluation were focused on, the element that most strongly correlated with palatability was taste, which was followed by texture and color, as found in Fig. 3. For each index, strong correlations were observed between the results of the sensory evaluation and those obtained using the sensing devices, as mentioned above. Nevertheless, the correlations between palatability and the results of the sensory evaluation were not in agreement with those between palatability and the results obtained using the sensing devices. One reason for this is considered to be the fact that the taste perceived by humans is affected by visual information. Hence, the interaction among indices perceived by different sensory organs should be taken into consideration to evaluate palatability by integrating multiple outputs from several sensing devices corresponding to the sensory organs of humans. Another reason is that the outputs from each sensing device are not necessarily in agreement with the quantified results of the sensory evaluation, even though the positional differences revealed using the sensing devices were in agreement with those obtained in the sensory evaluation. Multiple regression analysis was performed on the palatability in terms of the results of the physicochemical analyses of taste, texture and color. The obtained results agreed with the results obtained by the above analysis of the correlation between palatability and each index; i.e., the coefficient of each term in the multiple regression equation was highest for the $b$-value, as shown by 0.94 , whereas the magnitudes of other terms of koku 
and breaking stress at a strain of $50 \%$ were as low as 0.28 and 0.16 , respectively. The squared multiple correlation coefficient adjusted for the degrees of freedom $\left(R^{2}\right)$ was 0.95 . This result means that the palatability is mostly affected by the $b$-value (color), while the contributions from taste and texture are relatively low. This does not agree with the results of the sensory evaluations, as also discussed above. In-depth experiments and analyses are required to accurately quantify the palatability of food using sensing devices. We plan to carry out this task in our future research.

\section{Conclusion}

The aim of this study was to quantitatively determine the effects of different heating methods on positional differences in the palatability of simmered daikon samples. We carried out a sensory evaluation focusing on palatability and six other indices, and also performed physicochemical analyses using a taste sensor, a creep meter, and a colorimeter to examine indices corresponding to those examined in the sensory evaluation. The taste sensor was used to measure saltiness, umami, and koku, which were also examined in the sensory evaluation. For umami, the result obtained using the taste sensor was in agreement with that of the sensory evaluation. The creep meter was used to measure strain, which corresponded to the hardness examined in the sensory evaluation. The result of the physicochemical analysis agreed with that of the sensory evaluation. The colorimeter was used to analyze the color; for the $a$ - and $L$-values, the results were in agreement with those of the sensory evaluation. From the above, it was clarified that the difference in the heating method affects the degree of positional difference for simmered daikon samples. The positional differences in the palatability, taste, texture, and color of daikon samples subjected to pan surface heating (using a gas stove) were smaller than those of samples subjected to local heating (using an IH cooker).

The correlations between palatability and the indices of the sensory evaluation were not in agreement with those between palatability and the indices of the physicochemical analyses. Therefore, the interaction among the indices perceived by different sensory organs should be taken into consideration to objectively evaluate palatability using sensing devices. It is partly successful that the expression of the palatability was made using the multiple regression analysis in terms of physicochemical analyses of taste, texture and color. It is necessary to develop a technology for integrating various sensors and to extend the range of analysis targets beyond simmered food.

\section{References}

1 K. Toko, ed.: Biochemical Sensors, Mimicking Gustatory and Olfactory Senses (Pan Stanford Publishing, Singapore, 2014).

2 Y. Tahara and K. Toko: IEEE Sens. J. 13 (2013) 3001.

3 Y. Kobayashi, M. Habara, H. Ikezaki, R. Chen, Y. Naito and K. Toko: Sensors 10 (2010) 3411.

4 K. Toko, D. Hara, Y. Tahara, M. Yasuura and H. Ikezaki: Sensors 14 (2014) 16274. 
5 A. Riul, Jr., C. A. R. Dantas, C. M. Miyazaki and O. N. Oliveira, Jr.: Analyst 135 (2010) 2481.

6 D. Citterio and K. Suzuki: Anal. Chem. 80 (2008) 3965.

7 K. Toko: Sens. Actuators, B 64 (2000) 205.

8 K. Toko: Biomimetic Sensor Technology (Cambridge Univ. Press, Cambridge, U.K., 2000).

9 T. C. Pearce, S. S. Schiffman, H. T. Nagle and J. W. Gardner: Handbook of Machine Olfaction: Electronic Nose Technology (Wiley, New York, 2003).

10 T. Nakamoto: Human Olfactory Displays and Interfaces: Odor Sensing and Presentation (IGI Global, Hershey, 2012).

11 F. Röck, N. Barsan and U. Weimar: Chem. Rev. 108 (2008) 705.

12 J. Gardner and P. Bartlett: Sensors and Sensory Systems for an Electronic Nose (Kluwer, Boston, 1992).

13 M. Tatsuo, K. Kato, Y. Yamada and N. Ogawa: ITE Technical Report 35(33), ME2011-85 (2011) 23 (in Japanese).

14 Y. Fujimoto, H. Chiba, R. Okawa and K. Toko: Jpn. J. Taste Smell Res. 20 (2013) 317 (in Japanese).

15 H. Kawakami, Z. Tou, M. Fukuoka and N. Sakai: Jpn. J. Food Eng. 11 (2010) 51 (in Japanese).

16 Y. Ueda, M. Yonemitsu, T. Tsubuku, M. Sakaguchi and R. Miyajima: Biosci. Biotechnol. Biochem. 61 (1997) 1977.

17 A. K. M. Azad Shah, T. Ishihara, M. Ogasawara, H. Kurihara, N. Baba and K. Takahashi: Food Sci. Technol. Res. 16 (2010) 201.

18 T. Fukagawa, Y. Tahara, M. Yasuura, M. Habara, H. Ikezaki and K. Toko: J. Innov. Electron. Commun. 2 (2012) 1.

19 D. Hara, T. Fukagawa, Y. Tahara, M. Yasuura and K. Toko: Sens. Lett. 12 (2014) 1172.

20 M. Doi: Biochemical Sensors, Mimicking Gustatory and Olfactory Senses (K. Toko, ed., Pan Stanford Publishing, Singapore, 2014) Chap. 8.

21 T. Ito, K. Katsura and K. Iino: Jpn. J. Taste Smell Res. 4 (1997) 511 (in Japanese). 